\title{
Effects of Lansoprazole on the Lipopolysaccharide-Stimulated Toll-Like Receptor 4 Signal Transduction Systems: A Study Using the 293hTLR4/MD2-CD14 Cells
}

\author{
Tadashi Ohara ${ }^{1, *}$, Yuhsaku Kanoh², Keiichi Yoshino ${ }^{1}$, and Masaki Kitajima ${ }^{3}$ \\ 'Department of Gastroenterology, International University of Health and Welfare Hospital, \\ 537-3 Iguchi, Nasushiobara, Tochigi 329-2763, Japan \\ ${ }^{2}$ Department of Laboratory Medicine, Kitasato University, 1-15-1 Kitasato, Sagamihara, Kanagawa 228-8555, Japan \\ ${ }^{3}$ Center of Gastroenterology, International University of Health and Welfare Mita Hospital, \\ 1-4-3 Mita, Minato-Ku, Tokyo 108-8329, Japan
}

Received 27 April, 2009; Accepted 31 May, 2009

\begin{abstract}
Summary The present study was undertaken to evaluate the effects of lansoprazole (LPZ) on lipopolysaccharide (LPS)-stimulated toll-like receptor 4 (TLR4) signal transduction systems using the 293hTLR4/MD2-CD14 cells. The cells were incubated and then divided into the following groups: (a) untreated group, (b) non-LPZ treated (1h) group, (c) LPZ-treated (1h) plus non LPS-stimulated (1h) group, (d) LPZ-treated (1h) plus non LPS-stimulated (6h) group, (e) LPZ-treated (1h) plus LPS-stimulated (1h) group, (f) LPZ-treated (1h) plus LPS-stimulated (6h) group, (g) non LPZ-treated (1h) plus LPS-stimulated (1h) group and (h) non LPZ-treated (1h) plus LPS-stimulated (6h) group. Samples from each group were subjected to western blotting for analysis of IkB phosphorylation, intranuclear transfer of NF-kB, phosphorylation of MAP kinase (MAPK), intranuclear transfer of interferon regulatory factor 5 (IRF5), and expression of suppressor of cytokine signaling-1 (SOCS1). In the LPZ-treated groups, neither phosphorylation of MAPK nor intranuclear transfer of IRF5 was suppressed under stimulation with LPS, and enhanced intranuclear transfer of NF-kB and increased expression of SOCS1 were noted by comparison with the group treated with LPS alone. These results suggest that LPZ stimulates the expression of SOCS1 and regulates protein phosphorylation through its activity on TLR4 signal transduction under LPS stimulation.
\end{abstract}

Key Words: TLR4, lipopolysaccharide, lansoprazole, SOCS1, 293hTLR4/MD2-CD14 cell

\section{Introduction}

Toll-like receptors (TLRs) have been identified as a receptor of the innate immunity system which recognizes exogenous microorganisms $[1,2]$. Furthermore, analysis of gene-knockout mice demonstrated that TLRs regulates not only innate immunity, but also acquired immunity $[3,4]$. It

\footnotetext{
*To whom correspondence should be addressed.

Tel: +81-287-39-3060 Fax: +81-287-39-3001

E-mail: tohara@iuhw.ac.jp
}

has also been reported that excessive reactions of TLRs are involved in various diseases $[5,6]$. Toll-like receptor (TLR) 4 is a receptor capable of recognizing lipopolysaccharide (LPS). TLR4 signal transduction system is regulated the phosphorylation of IkB and NF-kB transfer into the nucleus, the phosphorylation of MAP kinase, interferon regulatory factor (IRF5) transfer into the nucleus. IRF5 transfer into the nucleus is concerned with the production of cytokines and regulates innate or acquired immunity no less than NF-kB [7-9]. Suppressor of cytokine signaling-1 (SOCS1) has been identified as a negative feed back gene involved in control of excessive LPS stimulation [10-13]. SOCS1 regulates the 
excess production of cytokines under stimulation with LPS. Proton pump inhibitor (PPI), which suppresses acid output, has been reported to additionally possess anti-inflammatory and immune actions $[14,15]$. The present study was undertaken to evaluate the effects of PPI on TLR4 signal transduction (protein phosphorylation) in the 293hTLR4/MD2CD14 cells (transfected with TLR4, MD2 and CD14 genes to yield human fibroblasts as HEK293 cells) under stimulation with LPS.

\section{Materials and Methods}

In order to study under the condition of similar living body, we used the 293hTLR4/MD2-CD14 cells in this study. The 293hTLR4/MD2-CD14 cells is a transfected cells with TLR4, MD2 and CD14 genes to yield HEK293 cell. We purchased the 293hTLR4/MD2-CD14 cells from InvivoGen (San Diego, CA). Lansoprazole (LPZ) as PPI was used. The additional concentration of LPZ to the cells was prepared as previously described [16]. The cells were incubated and aliquots of $1.5 \times 10^{6}$ cells were plated on collagen-coated 6-well culture plates (Falcon, Heidelberg, Germany) and cultured in 5\% $\mathrm{CO}_{2}$-supplemented room atmosphere. The viability of the 293 hTLR4/MD2-CD14 cells was more than $95 \%$ as assessed by trypan blue exclusion. And then, the cells divided into the following groups: (a) untreated group (no culture), (b) non LPZ-treated (1h) group, (c) LPZ $\left(10^{-4} \mathrm{M}\right)$-treated $(1 \mathrm{~h})$ plus non LPS-stimulated (1h) group, (d) LPZ $\left(10^{-4} \mathrm{M}\right)$-treated (1h) plus non LPS-stimulated (6 hours: 6h) group, (e) LPZ $\left(10^{-4} \mathrm{M}\right)$-treated (1h) plus LPS (1 $\mu \mathrm{g} / \mathrm{ml})$-stimulated (1h) group, (f) LPZ $\left(10^{-4} \mathrm{M}\right)$-treated (1h) pus LPS (1 $\mu \mathrm{g} / \mathrm{ml})$-stimulated (6h) group, (g) non LPZtreated $(1 \mathrm{~h})$ plus LPS $(1 \mu \mathrm{g} / \mathrm{ml})$-stimulated $(1 \mathrm{~h})$ group and (h) non LPZ-treated (1h) plus LPS $(1 \mu \mathrm{g} / \mathrm{ml})$-stimulated (6h) group. Extracts of samples from each group were subjected to the following analyses.

\section{Analysis of IkB phosphorylation and NF- $k B$ transfer into nucleus}

Phosphorylation of IkB was analyzed by western blotting using phospho-IkB- $\alpha$ (Ser32) antibody and IkB- antibody. NF-kB transfer into the nucleus was analyzed by western blotting of nuclear and cytoplasmic fractions using anti-NFkBp65 antibody. Phospho-IkB- $\alpha$ antibody, IkB- $\alpha$ antibody and anti-NF-kBp65 antibody were purchased from Santa Cruz Biotechnology (Santa Cruz, CA).

Analysis of phosphorylation of MAP kinase (MAPK: ERK, JNK and p38)

ERK was analyzed by western blotting using phosphop44/42 MAPK antibody and p44/42 MAPK antibody. JNK was analyzed by western blotting using phosphoSAPK/JNK (Thr183/Tyr185) antibody and SAPK/JNK
(56G8) rabbit mAb. P38 was analyzed by western blotting using phospho-p38MAPK (Thr180/Tyr182) antibody and p38MAPK antibody. Phospho-p44/42 MAPK antibody, p44/42 MAPK antibody, phospho-SAPK/JNK antibody, $\mathrm{SAPK} / \mathrm{JNK}$ rabbit $\mathrm{mAb}$ and $\mathrm{p} 38 \mathrm{MAPK}$ antibody were purchased from Santa Cruz biotechnology.

\section{Analysis of intranuclear transfer of IRF5}

For analysis of IRF5 transfer into the nucleus, the nuclear and cytoplasmic fractions were exposed to the primary antibody (anti-IRF5 antibody) and the HRP-labeled secondary antibody (anti-goat IgG-HRP), followed by western blotting. Anti-IRF5 antibody and anti-gout IgG-HRP were purchased from Santa Cruz Biotechnology.

\section{Analysis of SOCS1 expression}

For analysis of SOCS1 expression, samples were exposed to the primary antibody (anti-SOCS1 antibody) and the HRP-labeled secondary antibody (anti-rabbit IgG-HRP), followed by western blotting. Anti-SOCS1 antibody and anti-rabbit IgG-HRP were purchased from Santa Cruz Biotechnology.

\section{Western blot analysis}

At the end of the incubation period, the medium was removed and the cells were immediately lysed and transferred by sodium dodecyl sulfate/polyacrylamide gel electrophoresis; proteins were then blotted to nitrocellulose membranes using a semidry transfer apparatus (Pharmacia Biotech, Freiburg, Germany). Blots were blocked for $2 \mathrm{~h}$ in $5 \%$ (wt/vol) bovine serum albumin containing $20 \mathrm{mmol} / \mathrm{L}$ Tris, $\mathrm{pH} \mathrm{7.5,} 150 \mathrm{mmol} / \mathrm{L} \mathrm{NaCl}$, and $0.1 \%$ Tween 20 (TBS-T) and then incubated at $4{ }^{\circ} \mathrm{C}$ overnight with the first antibody. Following washing with TBS-T and incubation with horseradish peroxidase-coupled anti-mouse, anti-goat, or anti-rabbit immunoglobulin $\mathrm{G}$ antibody at room temperature for $2 \mathrm{~h}$, respectively, the blots were washed extensively and developed using enhanced chemiluminescent detection (Amersham Pharmacia Biotech, Uppsala, Sweden). Blots were exposed to Kodak X-OMAT AR-5 film (Eastman Kodak Co., Rochester, NY).

\section{Results}

In the LPS-stimulated group, the phosphorylation of IkB, phospho-p44/42 MAPK antibody and p44/42 MAPK antibody, phospho-SAPK/JNK (Thr183/Tyr185) antibody) and phospho-p38MAPK (Thr180/Tyr182) antibody and p38MAPK antibody was suppressed after $6 \mathrm{~h}$ of stimulation as compared to that observed after $1 \mathrm{~h}$ of stimulation (Figs. 1, 2, 3 and 4), and intranuclear transfer of IFR5 and NF-kB was noted (Figs. 5 and 6). In the LPZ-treated group, the phosphorylation of IkB, phospho-p44/42 MAPK antibody 
$\begin{array}{llllllll}1 & 2 & 3 & 4 & 5 & 6 & 7 & 8\end{array}$

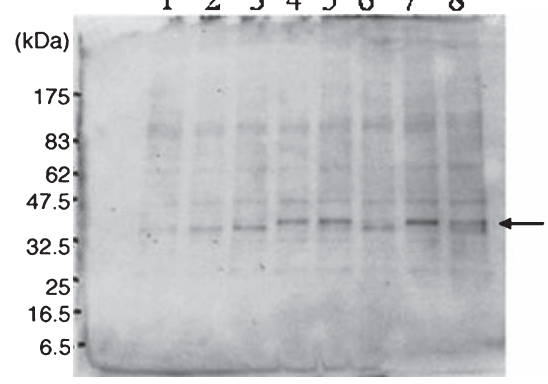

Anti-phospho-IkBa

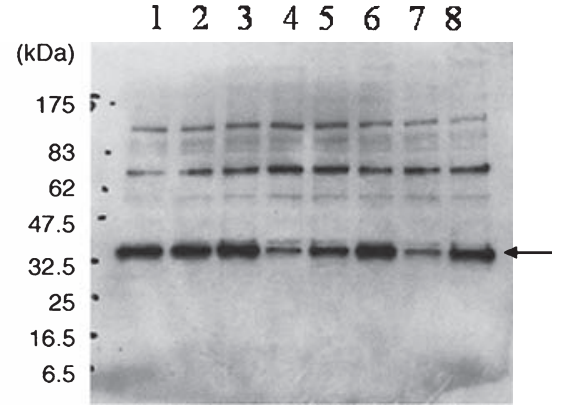

Anti-IkBa

1. +LPZ(lh), - LPS

2. + LPZ(lh), - LPS (lh)

5. $+\mathrm{LPZ}(\mathrm{lh}),+\mathrm{LPS}(6 \mathrm{~h})$

3. + LPZ(lh), - LPS $(6 h)$

6. - LPZ(lh)

4. +LPZ(lh), +LPS(lh)

7. - LPZ(lh), +LPS(lh)

8. - LPZ(lh), +LPS $(6 \mathrm{~h})$

Fig. 1. The phosphorylation of IkB- $\alpha$ analyzed by western-blotting. An arrow indicates the induction of IkB- $\alpha$. LPZ and LPS denotes lansoprazole and lipopolysaccharide, respectively.
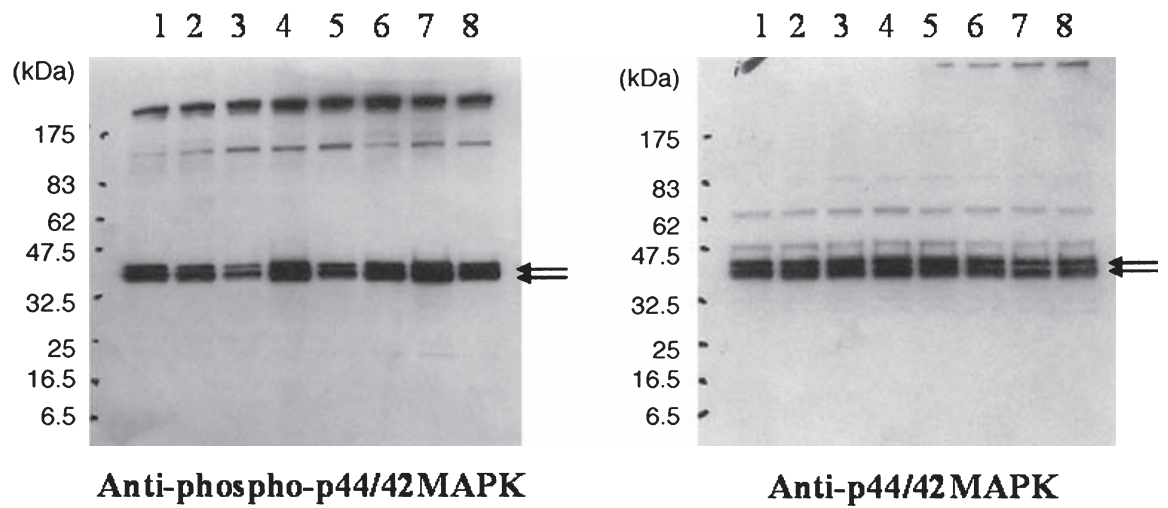
1. +LPZ(lh), - LPS
2. +LPZ(lh), - LPS(lh)
3. $+\mathrm{LPZ}(\mathrm{lh}),-\mathrm{LPS}(6 \mathrm{~h})$
4. $+\mathrm{LPZ}(\mathrm{lh}),+\mathrm{LPS}(\mathrm{lh})$

5. $+\mathrm{LPZ}(\mathrm{lh}),+\mathrm{LPS}(6 \mathrm{~h})$

6. - LPZ(lh)

7. - LPZ(lh), +LPS(lh)

8. - LPZ(lh), +LPS (6h)

Fig. 2. The phosphorylation of ERK (phospho-p44/42 MAPK antibody and p44/42 MAPK antibody) analyzed by western-blotting. Arrows indicate the induction of phospho-p44/42 MAPK antibody and p44/42 MAPK antibody, respectively.

and p44/42, phospho-SAPK/JNK (Thr183/Tyr185) antibody) and phospho-p38MAPK (Thr180/Tyr182) antibody and p38MAPK antibody was not suppressed (Figs. 1, 2, 3 and 4), and intranuclear transfer of IFR5 was not suppressed under stimulation with LPS (Fig. 6). The induction of SAPK/JNK (56G8) rabbit $\mathrm{mAb}$ was yet presence in all groups (Fig. 3). And then in the LPZ-treated groups, enhanced intranuclear transfer of NF-kB and increased expression of SOCS1 were noted, as compared to the observations in the group stimu- lated with LPS alone (Fig. 7).

\section{Discussion}

The present study was undertaken to examine whether or not LPZ might suppress phosphorylation of IkB and MAPK or intranuclear transfer of NF-kB and IRF5 and to evaluate the effects of LPZ on SOCS1 as a negative feedback gene involved in the TLR4 signal transduction system under 
$\begin{array}{llllllll}12 & 3 & 4 & 5 & 6 & 7 & 8\end{array}$

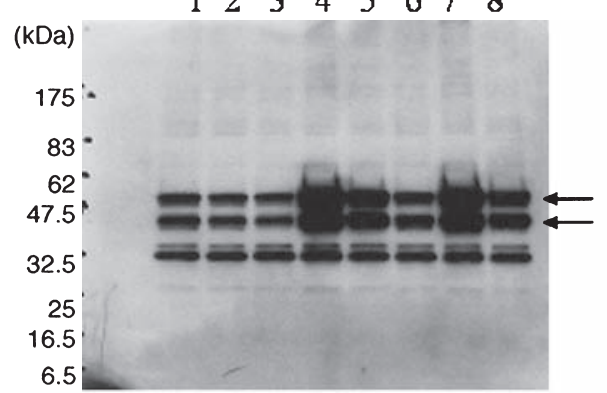

Anti-phospho-SAPK/JNK $\begin{array}{llllllll}1 & 2 & 3 & 4 & 5 & 6 & 7 & 8\end{array}$

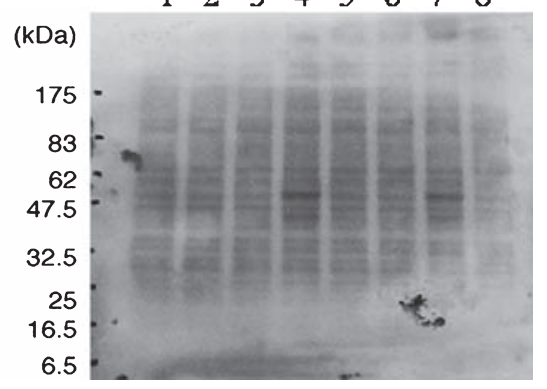

Anti-SAPK/JNK
1. +LPZ(lh), - LPS
5. $+\mathrm{LPZ}(\mathrm{lh}),+\mathrm{LPS}(6 \mathrm{~h})$
2. +LPZ(lh), - LPS(lh)
6. - LPZ(lh)
3. +LPZ(1h), - LPS (6h)
7. - LPZ(lh), +LPS(lh)
4. $+\mathrm{LPZ}(\mathrm{lh}),+\mathrm{LPS}(\mathrm{lh})$
8. - LPZ(lh), +LPS $(6 h)$

Fig. 3. The phosphorylation of JNK (phospho-SAPK/JNK (Thr183/Tyr185) antibody and SAPK/JNK (56G8) rabbit mAb) analyzed by western-blotting. Arrows indicate the induction of phospho-SAPK/JNK (Thr183/Tyr185) antibody. The phosphorylation of $\mathrm{SAPK} / \mathrm{JNK}$ (56G8) rabbit mAb was obscure.

$\begin{array}{llllllll}1 & 2 & 3 & 4 & 5 & 6 & 7 & 8\end{array}$

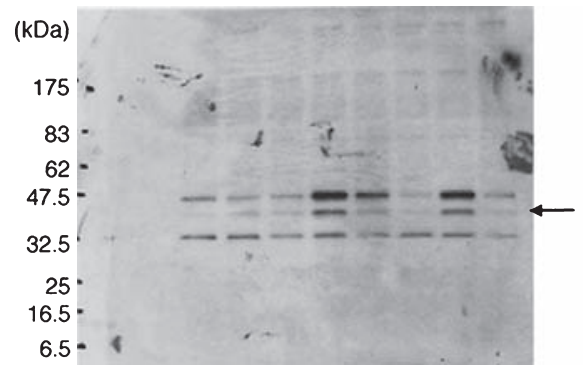

Anti-phospho-p38MAPK $\begin{array}{llllllll}1 & 2 & 3 & 4 & 5 & 6 & 7 & 8\end{array}$

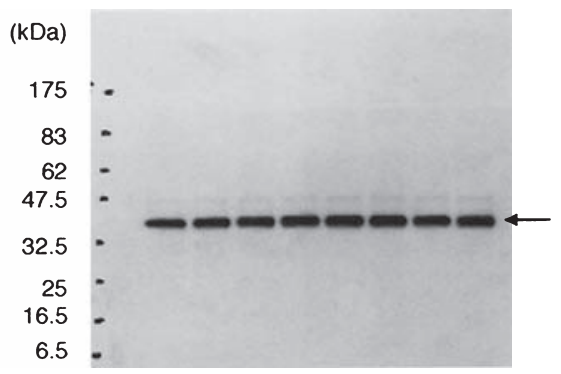

Anti-p38MAPK

$$
\begin{aligned}
& \text { 1. +LPZ(lh), - LPS } \\
& \text { 2. +LPZ(lh), - LPS }(1 h) \\
& \text { 3. +LPZ(lh), - LPS }(6 h) \\
& \text { 4. +LPZ(lh), +LPS }(1 h)
\end{aligned}
$$$$
\text { 5. }+\mathrm{LPZ}(1 \mathrm{~h}),+\mathrm{LPS}(6 \mathrm{~h})
$$$$
\text { 6. - LPZ(lh) }
$$$$
\text { 7. - LPZ(lh), +LPS(lh) }
$$$$
\text { 8. - LPZ(lh), +LPS }(6 h)
$$

Fig. 4. The phosphorylation of p38 (phospho-p38MAPK (Thr180/Tyr182) antibody and p38MAPK antibody) analyzed by westernblotting. An arrow indicates the induction of phospho-p38MAPK (Thr180/Tyr182) antibody and p38MAPK antibody, respectively.

stimulation with LPS. In the analysis of the effects of LPZ on LPS-stimulated TLR4 signal transduction, LPZ did not suppress either phosphorylation of MAPK (IkB, ERK, JNK or p38) or IRF5 transfer into the nucleus. NK-kB transfer into the nucleus, which might possibly lead to exacerbation of inflammatory reactions, seemed to be stimulated by LPS. In the LPZ-treated group, increase in the expression of
SOCS1 was observed. Previous studies using various systems have demonstrated that LPZ exerts anti-inflammatory activity such as the suppression of cytokine formation. Taking into consideration the results of the present study and previous reports, it seems possible that LPZ stimulates the transfer of NF-kB into the nucleus during LPS-stimulated TLR4 signal transduction, which, in turn, leads to the induc- 
12345678

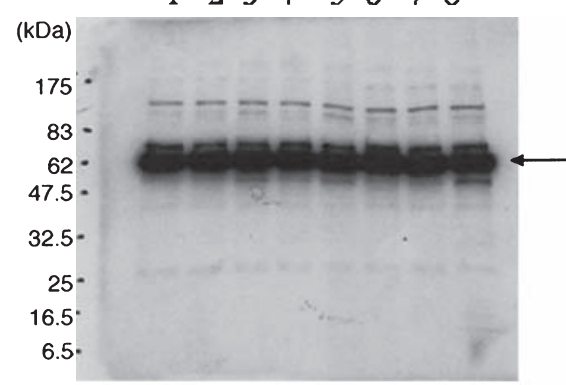

Anti-NF-kBp65 of cytoplasm

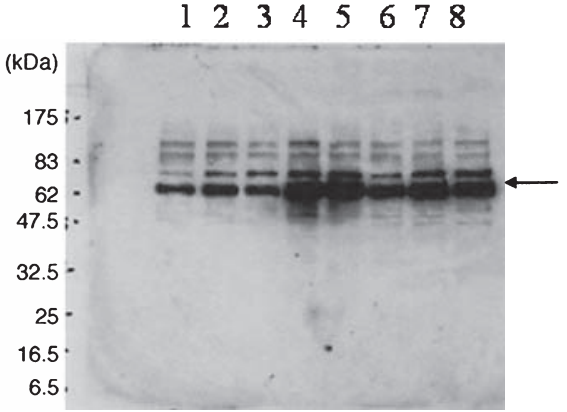

Anti-NF-kBp65 of nuclear ex tracts
1. +LPZ(lh), - LPS
5. $+\mathrm{LPZ}(\mathrm{lh}),+\mathrm{LPS}(6 \mathrm{~h})$
2. +LPZ(lh), - LPS(lh)
6. - LPZ(lh)
3. $+\mathrm{LPZ}(\mathrm{lh}),-\mathrm{LPS}(6 \mathrm{~h})$
7. - LPZ(lh), +LPS(lh)
4. $+\mathrm{LPZ}(\mathrm{lh}),+\mathrm{LPS}(\mathrm{lh})$
8. - LPZ(lh), +LPS (6h)

Fig. 5. The result of the transition from cytoplasm to nucleus of NF-kBp65 analyzed by western-blotting. An arrow indicates the induction of NF-kB.
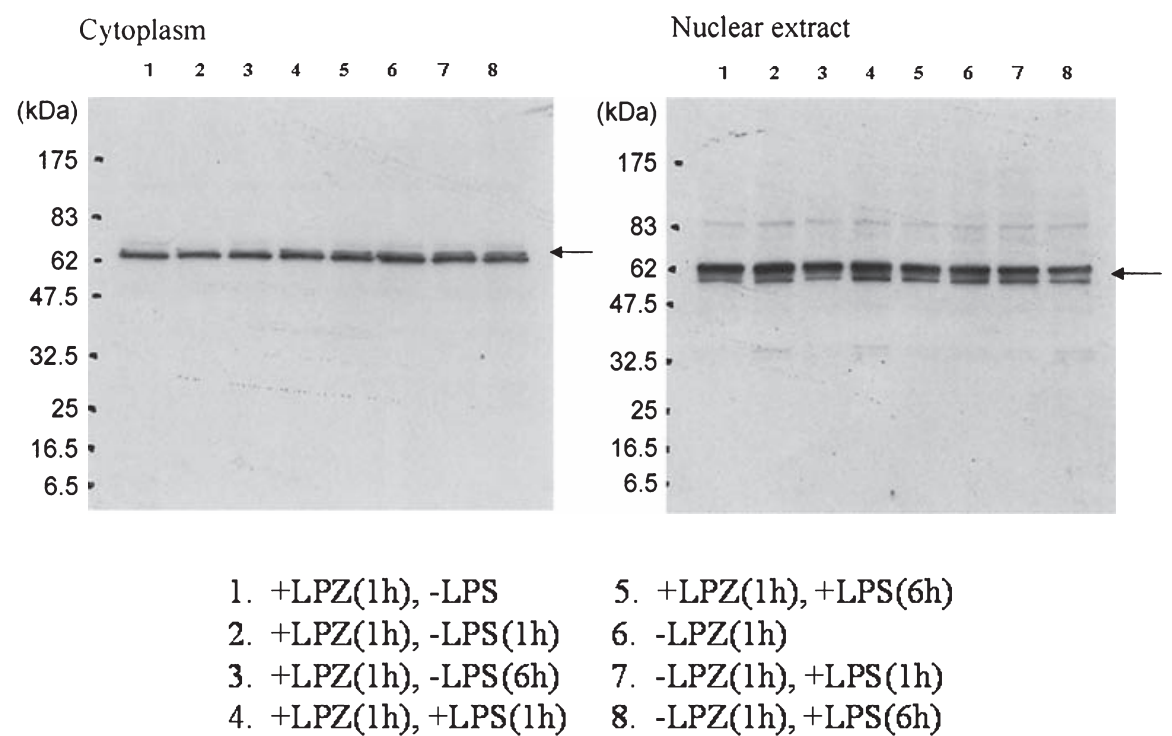

Fig. 6. The result of the transition from cytoplasm to nucleus of IRF5 analyzed by western-blotting. An arrow indicates the induction of IRF5.

tion of SOCS1 expression and anti-inflammatory activity. Because of limitations related to cell specificity, the results of this study cannot be reasonably extrapolated to other cells. For strict demonstration of the anti-inflammatory activity of LPZ using the test system employed in this study, it is necessary to measure the cytokines formed in both the LPZ-treated groups and non LPZ-treated groups. Furthermore, since cell extracts were used in the present study, it remains unknown whether the results from this study might reflect the events in the cell membrane or the events in the cytoplasm. It has recently been reported that MyD88, an adaptor molecule for TLRs (TLR2, 4), regulates the conduction of stimuli through TLRs, working together with heat shock protein (HSP)-associated molecules, such as HSP70, HSP90, Grp96 and Grp78 [17-21]. Also for the testing system employed in this study, it would be desirable to clarify the involvement of these HSP-associated molecules. In any event, the results of the present study suggest the possibility that LPZ induces SOCS1 and this regulates protein phosphorylation during TLR4 signal transduction. 


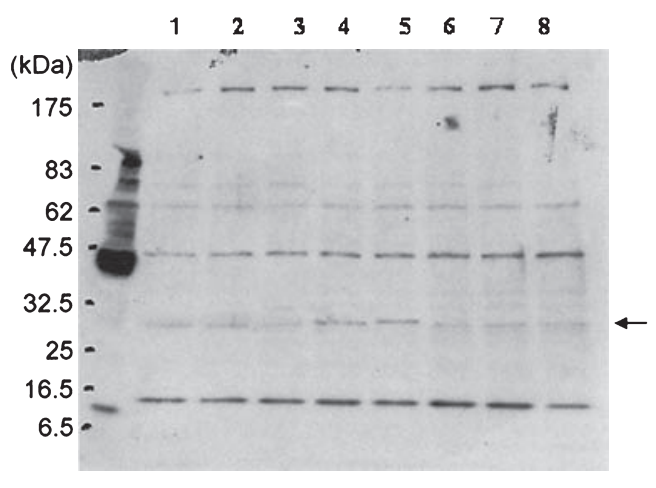

$$
\begin{array}{ll}
\text { 1. }+\operatorname{LPZ}(1 \mathrm{~h}),-\operatorname{LPS} & \text { 5. }+\mathrm{LPZ}(1 \mathrm{~h}),+\mathrm{LPS}(6 \mathrm{~h}) \\
\text { 2. }+\mathrm{LPZ}(1 \mathrm{~h}),-\operatorname{LPS}(1 \mathrm{~h}) & \text { 6. }-\operatorname{LPZ}(1 \mathrm{~h}) \\
\text { 3. }+\mathrm{LPZ}(1 \mathrm{~h}),-\operatorname{LPS}(6 \mathrm{~h}) & \text { 7. }-\operatorname{LPZ}(1 \mathrm{~h}),+\mathrm{LPS}(1 \mathrm{~h}) \\
\text { 4. }+\mathrm{LPZ}(1 \mathrm{~h}),+\mathrm{LPS}(1 \mathrm{~h}) & \text { 8. }-\operatorname{LPZ}(1 \mathrm{~h}),+\mathrm{LPS}(6 \mathrm{~h})
\end{array}
$$

Fig. 7. The expression of SOCS1 analyzed by western-blotting. An arrow indicates the induction of SOCS1.

\section{Conclusion}

These results suggest that LPZ stimulates the expression of SOCS1 and regulates protein phosphorylation through its activity on TLR4 signal transduction under LPS stimulation in the 293 hTLR4/MD2-CD14 cells. This is a noteworthy new finding. We propose to analysis the particular account of this study using different cell lines or using cell cultures other techniques.

\section{Acknowledgments}

This work was supported by research subsidies from Takeda Pharmaceutical Industry Corporation, Japan. The sponsor of the study had no role in study design, data collection, data analysis, data interpretation, or writing of the report. The corresponding author had full access to all the data in the study and had final responsibility for the decision to submit for publication.

\section{Abbreviations}

LPZ, Lansoprazole; LPS, Lipopolysaccharide; TLRs, Tolllike receptors; MAPK, MAP kinase; IRF5, Interferon regulatory factor 5; SOCS1, Suppressor of cytokine signaling-1; PPI, Proton pump inhibitor; HSP, Heat shock protein.

\section{References}

[1] Akira, S., Takeda, K., and Kaisho, T.: Toll-like receptors: critical proteins linking innate and acquired immunity. Nat.
Immunol., 2, 675-680, 2001.

[2] Takeuchi, O., Hoshino, K., and Akira, S.: TLR 2-deficient and MyD88-deficient mice are highly susceptible to Staphylococcus aureus infection. J. Immunol., 165, 53925396, 2000.

[3] Diebold, S.S.: Activation of dendritic cells by toll-like receptors and C-type lectins. Handb. Exp. Pharmacol., 188, 3-30, 2009.

[4] Goodridge, F.S., Shimada, T., Wolf, A.J., Hsu, Y.M., Becker, C.A., Lin, X., and Underhill, D.M.: Differential use of CARD9 by Dectin-1 in macrophages and dendritic cells. $J$. Immunol., 182, 1146-1154, 2009.

[5] Beutler, B.: Microbe sensing, positive feedback loops, and the pathogenesis of inflammatory diseases. Immunol. Rev., 227, 248-263, 2009.

[6] Bochud, P.Y., Chien, J.W., Marr, K.A., Leisenring, W.M., Upton, A., Janer, M., Rodrigues, S.D., Li, S., Hansen, J.A., Zhao, L.P., Adererm, A., and Boeckn, M.: Toll-like receptor 4 polymorphisms and aspergillosis in stem-cell transplantation. N. Eng. J. Med., 359, 1766-1777, 2008.

[7] Richez, C., Tasuda, K., Watkins, A.A., Akira, S., Lafyatis, R., van Seventer, J.M., and Rifkin, I.R.: TLR4 ligands induce IFN-alpha production by mouse conventional dendritic cells and human monocytes after IFN-beta priming. J. Immunol., 182, 820-828, 2009.

[8] Paun, A., Reiner, J.T., Jiang, Z., Medin, C., Baikhi, M.Y., Fitzgerald, K.A., and Piha, P.M.: Functional characterization of murine interferon regulatory factor 5 (IRF5) and its role in the innate antiviral response. J. Boil. Chem., 283, 1429514308, 2008.

[9] O'Neill, L.A. and Bowie, A.G.: The family of five: TIRdomain-containing adaptors in Toll-like receptor signaling. Nat. Rev. Immunol., 7, 353-364, 2007.

[10] Mostecki, J., Showalter, B.M., and Rothman, P.B.: Early growth response-1 regulate lipopolysaccharide-induced suppressor of cytokine signaling-1 transcription. J. Biol. Chem., 280, 2596-2605, 2005.

[11] Prele, C.M., Woodward, E.A., Bisley, J., Keith-Magee, A., Nicholson, S.E., and Hart, P.H.: SOCS1 regulates the IFN but not NF kappa B pathway in TLR-stimulated human monocytes and macrophages. J. Immunol., 181, 8018-8026, 2008.

[12] Mansell, A., Smith, R., Doyle, S.L., Gray, P., Fenner, J.E., Crack, P.J., Nicholson, S.E., Hilton, D.J., O’Neil, L.A., and Hertzog, P.J.: Suppressor of cytokine signaling 1 negatively regulates Toll-like receptor signaling by mediating Mal degradation. Nat. Immunol., 7, 148-155, 2006.

[13] Adib-Conquy, M., Adrie, C., Fitting, C., Gattolliat, O., Beyaert, R., and Cavaillon, J.M.: Up-regulation of MyD88s and SIGIRR, molecules inhibiting Toll-like receptor signaling, in monocytes from septic patients. Crit. Care Med., 34, 2377-2385, 2006.

[14] Handa, O., Yoshida, N., Fujita, N., Tanaka, Y., Ueda, M., Takagi, T., Kokura, S., Naito, Y., Okanoue, T., and Yoshikawa, T.: Molecular mechanisms involved in anti-inflammatory effects of proton pump inhibitors. Inflamm. Res., 55, 476480, 2006. 
[15] Kuroda, M., Yoshida, N., Ichikawa, H., Takagi, T., Okuda, T., Naito, Y., Okanoue, T., and Yoshikawa, T.: Lansoprazole, a proton pump inhibitor, reduces the severity of indomethacininduced rat enteritis. Int. J. Mol. Med., 17, 89-93, 2006.

[16] Yoshida, N., Yoshikawa, T., Tanaka, N., Kasai, Y., Naito, Y., and Kondo, M.: A new mechanism for anti-inflammatory actions of proton pump inhibitors-inhibitory effects on neutrophil-endotherial cell interactions. Aliment. Pharmacol. Ther., 14 Suppl. 1, 74-81, 2000.

[17] Luo, X., Zuo, X., Zhang, B., Song, L., Wei, X., Zhou, Y., and Xiao, X.: Release of heart shock protein 70 and the effects of extracellular heart shock protein 70 on the production of IL-10 in fibroblast-like synoviocytes. Cell Stress Chaperones, 13, 365-373, 2008.
[18] Asea, A.: Heat shock proteins and toll-like receptors. Hndb. Exp. Pharmacol., 183, 111-127, 2008.

[19] Warger, T., Hilf, N., Rechtsteiner, G., Haselmayer, P., Carrick, D.M., Jonuleit, H., von Landenberg, P., Rammensee, H.G., Nicchitta, C.V., Radak, M.P., and Schild, H.: Interaction of TLR2 and TLR4 ligands with the N-terminal domain of Gp96 amplifies innate and adaptive immune responses. J. Biol. Chem., 281, 22545-22553, 2006.

[20] Osterioh, A. and Breloer, M.: Heart shock proteins: linking danger and pathogen recognition. Med. Microbiol. Immunol., 197, 1-8, 2008.

[21] Brown, V., Brown, R.A., Ozinsky, A., Hesselberth, J.R., and Fields, S.: Binding specificity of Toll-like receptor cytoplasmic domains. Eur. J. Immunol., 36, 742-753, 2006. 